\title{
On the representation of metric spaces
}

\author{
G.J. Logan
}

A closure algebra is a set $X$ with a closure operator $C$ defined on it. It is possible to construct a topology $\tau$ on $M_{X}$, the family of maximal, proper, closed subsets of $X$, and then to examine the relationship between the algebraic structure of $(X, C)$ and the topological structure of the dual space $\left(M_{X}, \tau\right)$. This paper describes the algebraic conditions which are necessary and sufficient for the dual space to be separable metric and metric respectively.

In Logan [3] a method was given for representing any $T_{1}$-space as a particular kind of dual space of a closure algebra. The present paper improves the manner in which the closure algebra is constructed, and specializes the result to separable metric spaces and to metric spaces in general.

The results seem to be of some interest because they offer a characterization of metric and separable metric spaces which to my knowledge is completely new.

The notation, results, and references in Logan [3], [4] are presupposed: in particular if $(X, C)$ is a closure algebra, then $M_{X}$ is the family of maximal consistent sets; $S: P(X) \rightarrow P\left(M_{X}\right)$, defined by $S(A)=\left\{\Delta \in H_{X}: A \subseteq \Delta\right\}$ will satisfy $A \subseteq B \Rightarrow S(B) \subseteq S(A)$, and 
$\mathscr{L}\left(\bigcup_{k \in K} A_{k}\right)=\bigcap_{k \in K} S\left(A_{k}\right)$ for each $\left\{A_{k}\right\}_{k \in K} \subseteq P(X) ;$ and $B=\left\{S\left(A_{f}\right): A_{f} \subseteq X, A_{f}\right.$ finite $\}$ will be the base for a topology $\tau$ on the dual space $\left(M_{X}, \tau\right)$. In addition the closure algebra will be said to be countable precisely when $X$ is a countable set.

The following definitions will be required.

(i) Let $(X, C)$ be a closure algebra, $\Delta$ a maximal consistent subset of $X$, and $\xi$ a family of maximal consistent subsets; $\Delta$ is said to be separated from $\xi$ if $\Delta$ has some fixed finite subset that is inconsistent with some finite subset of each member of $\xi$ ( $A$ is said to be inconsistent with $B$ if and only if $C(A \cup B)=X)$. If from the members of $\xi$ together there are only finitely many finite subsets that are consistent with some fixed finite subset of $\Delta$ then $\Delta$ is said to be strongly separated from $\xi$.

(ii) The closure algebra is said to be separated (strongly separated) if for each maximal consistent set $\Delta$ and each $A_{f}$ a finite subset of $\Delta, \Delta$ is separated (strongly separated) from the family of maximal consistent sets that do not contain $A_{f}$ (from now on I use $A_{f}, A_{g}, A_{h}, A_{k}$ to denote finite subsets of $X$ ).

In order to indicate that the terminology is not misleading I now show that every strongly separated closure algebra is separated. Suppose that $A_{f} \subseteq \Delta \in M_{X}$ and that $(X, C)$ is strongly separated. There is some $A_{g} \subseteq \Delta$ and the family

$$
\Gamma=\left\{A_{h}: C\left(A_{g} \cup A_{h}\right) \neq X, A_{h} \subseteq \Delta_{0} \text { for some } \Delta_{0} \text { with } A_{f} \pm \Delta_{0}\right\}
$$

is finite. If $A_{f} \nsubseteq \Delta_{I}$ and $\Delta_{1}$ has more than a finite number of finite subsets then there is some finite subset of $\Delta_{I}$ that is not a member of $\Gamma$ and so is inconsistent with $A_{g}$ and thus is also inconsistent with $A_{f} \cup A_{g}$ * On the other hand if $\Delta_{1}$ has only a finite number of finite subsets then $\Delta_{1}$ is finite. Since $A_{f} \nsubseteq \Delta_{1}, A_{f} \cup A_{g} \nsubseteq \Delta_{1}$, and so $C\left(\left(A_{f} \cup A_{g}\right) \cup \Delta_{1}\right)=X$ (otherwise the maximality of $\Delta_{1}$ is contradicted). 
In either case $A_{f} \cup A_{g}$ is a finite subset of $\Delta$ that is inconsistent with some finite subset of each $\Delta_{1}$ that satisfies $A_{f} \pm \Delta_{l}$; that is, $(X, C)$ is separated.

THEOREM 1. If $(X, C)$ is a countable, separated closure algebra, then the dual space $\left(M_{X}, \tau\right)$ is a separable metric space.

Proof. On account of Urysohn's Theorem (see Kelley [2], p. 125) it is only necessary to show that $\left(M_{X}, \tau\right)$ is a regular $T_{I}$-space with a countable base. That $\left(M_{X}, \tau\right)$ is $T_{1}$ is proved in Logan [3], and it is clear that the base $B=\left\{S\left(A_{f}\right): A_{f} \subseteq X\right\}$ is countable since the family of finite subsets of the countable set $X$ is countable.

To see that $\left(M_{X}, \tau\right)$ is regular, suppose that $Q$ is a closed subset of $M_{X}$ and $\Delta \in M_{X} \backslash Q$. Then there is some $S\left(A_{f}\right) \in B$ with $\Delta \in S\left(A_{f}\right)$ and $S\left(A_{f}\right) \cap Q=\emptyset$. Let $k \in K$ index the members of $Q$; then $A_{f} \subseteq \Delta$ and $A_{f} \pm \Delta_{k}$ for each $k \in K$. Since $(X, C)$ is separated we may choose $A_{g} \subseteq \Delta$, so that for each $k \in K$ there is some $A_{k} \subseteq \Delta_{k}$ with $C\left(A_{g} \cup A_{k}\right)=X$. But now, for each $k \in K$,

$$
S\left(A_{g}\right) \cap S\left(A_{k}\right)=S\left(A_{g} \cup A_{k}\right)=\varnothing
$$

and so

$$
S\left(A_{g}\right) \cap \underset{k \in K}{U} S\left(A_{k}\right)=\emptyset
$$

Since each $\Delta_{k} \in Q$ satisfies $\Delta_{k} \in S\left(A_{k}\right)$, we have that $\varrho \subseteq \underset{k \in K}{U} S\left(A_{k}\right)$, which is open in ${ }^{\prime} X$. On the other hand $\Delta \in S\left(A_{g}\right)$, which is open in $M_{X}$, so that $\left(H_{X}, \tau\right)$ is regular. //

The question arises naturally as to whether every separable metric space may be considered to be the dual space of a suitable closure algebra. Theorem 2 shows that every separable metric space may be represented as the dual space of a closure algebra satisfying the conditions given in Theorem 1 . 
THEOREM 2. Every separable metric space is homeomorphic to the dual space of a countable, separated closure algebra.

Proof. Let $Y$ be a separable metric space; then by Urysohn's Theorem $Y$ is a regular $T_{1}$-space with a countable base, and consequently a space with a countable subbase. Let this countable subbase be, $X$ and define a function $C: P(X) \rightarrow P(X)$ by $C(A)=\left\{u \in X: \bigcap_{v \in A} v \subseteq u\right\}$. It is easily verified that $(X, C)$ is a closure algebra. For each $y \in Y$ define $\Gamma_{y}=\{u \in X: y \in u\}$; then $\Gamma_{y}$ is closed with respect to $c$, since if $u \in C\left(\Gamma_{y}\right)$ then $u \supseteq \prod_{v \in \Gamma_{y}} v \supseteq\{y\}$, and so $u \in \Gamma_{y}$.

Furthermore, each $\Gamma_{y}$ is consistent. For if we choose $y_{1} \in Y, y_{1} \neq y$, then there is some nember of the base that contains $y_{1}$ and not $y$ ( $y$ is $T_{1}$ ), and so there is some $u \in X$ with $y_{1} \in u$ and $y \notin u$. Since $u \notin \Gamma_{y}=C\left(\Gamma_{y}\right)$ we have that $C\left(\Gamma_{y}\right) \neq X$.

We also have that each $\Gamma_{y}$ is maximal consistent. For if $u_{1} \in X \backslash \Gamma_{y}$ then $y \notin u_{1}$, and for each $y_{1} \in u_{1}$ there is some $v \in X$ with $y \in v$, $y_{1} \notin v\left(Y\right.$ is $\left.T_{1}\right)$. We may infer that each $y_{1} \in u_{1}$ is not a member of $\prod_{v \in \Gamma_{y}} v$ (since $y \in v$ implies $v \in \Gamma_{y}$ ) and that $u_{I} \cap \bigcap_{v \in \Gamma_{y}} v=\varnothing$. But now

$$
\begin{aligned}
C\left(\Gamma_{y} \cup\left\{u_{1}\right\}\right) & =\left\{u: \cap\left\{v: v \in \Gamma_{y} \cup\left\{u_{1}\right\}\right\} \subseteq u\right\} \\
& =\left\{u: u_{1} \cap \cap_{v \in \Gamma_{y}} v \subseteq u\right\} \\
& =\{u: \emptyset \subseteq u\} \\
& =X
\end{aligned}
$$

so that $\Gamma_{y} \cup\left\{u_{1}\right\}$ is inconsistent and $\Gamma_{y}$ is maximal consistent. This shows that $\left\{\Gamma_{y}: y \in Y\right\} \subseteq M_{X}$.

For the opposite inclusion, if $\Delta \in M_{X}$ then the consistency of $\Delta$ 
ensures that $\bigcap_{u \in \Delta} u \neq \varnothing$. Choose $y \in \underset{u \in \Delta}{U} u$; then $\bigcap_{u \in \Gamma_{y}} u \subseteq \bigcap_{u \in \Delta} u$, and by the definition of $C, C\left(\Gamma_{y}\right) \subseteq C(\Delta)$. But $\Gamma_{y}, \Delta$ are both closed with respect to $C$ and so $\Gamma_{y} \subseteq \Delta$. Since $\Delta$ is maximal, $\Gamma_{y}=\Delta$, and so each maximal consistent set has the form $\Gamma_{y}$ for some $y \in Y$. This shows that $\left\{\Gamma_{y}: y \in Y\right\}=M_{X}$.

To see that $M_{X}$ and $Y$ are homeomorphic, define $\theta: Y \rightarrow M_{X}$ by $\theta(y)=\Gamma_{y}$, for each $y \in Y$. Clearly $\theta$ is an onto function, and $\theta$ is one-to-one since if $y_{1} \neq y_{2}$ then we may choose $u \in X$ such that $y_{1} \in u, y_{2} \notin u$, and so $u \in \Gamma_{y_{1}}, u \notin \Gamma_{y_{2}}$, yielding that $\theta\left(y_{1}\right) \neq \theta\left(y_{2}\right)$.

$\theta$ is continuous, since if $0 \in \tau$ then $\theta^{-1}[0]=\theta^{-1}\left[\bigcup_{f \in F} S\left(A_{f}\right)\right]$, where $\left\{S\left(A_{f}\right)\right\}_{f \in F}$ is a subfamily of the base for $\tau$,

$$
\begin{aligned}
& =\underset{f \in F}{U} \theta^{-1}\left[S\left(A_{f}\right)\right] \\
& =\underset{f \in F}{U} \theta^{-1}\left[S\left(\underset{u \in A_{f}}{U}\{u\}\right)\right] \\
& =\bigcup_{f \in F} \theta^{-1}\left[\prod_{u \in A_{f}} s(u)\right] \\
& =\underset{f \in F}{u}\left(\prod_{u \in A_{f}} \theta^{-1}[S(u)]\right) \\
& =\cup_{f \in F}\left(\bigcap_{u \in A_{f}} \theta^{-1}\left\{\Delta \in M_{X}: u \in \Delta\right\}\right) \\
& =u_{f \in F}\left(\bigcap_{u \in A} \theta^{-1}\left\{\Gamma_{y}: y \in u\right\}\right)
\end{aligned}
$$




$$
\begin{aligned}
& =\underset{f \in F}{U}\left(\bigcap_{\mathcal{u} \in A_{f}}\{y: y \in u\}\right) \\
& =\underset{f \in F}{U}\left(\bigcap_{u \in A_{j}} u\right) .
\end{aligned}
$$

Now for each $f \in F, A_{f}$ is finite and each $u$ is a member of the subbase for $Y$. Hence each $\bigcap_{u \in A_{f}} u$ is a member of the base and so $\theta^{-1}[0]$ is open in $y$.

Furthermore $\theta^{-1}$ is continuous, since if $P$ is open in $Y$, then
$U_{k \in K}\left(\begin{array}{cc}n & u \\ u \in F_{k}\end{array}\right)$ where each $u \in X$, and for each $k \in K, F_{k}$ is finite. Hence

$$
\begin{aligned}
& \theta(P)=\bigcup_{k \in K} \theta\left(\bigcap_{u \in F_{k}} u\right) \\
& \left.=\underset{k \in K}{\bigcup} \prod_{u \in F_{k}} \theta(u)\right) \\
& =\underset{k \in K}{u}\left\{\bigcap_{u \in F_{k}}\left\{\Gamma_{y}: y \in u\right\}\right\} \\
& \left.={\underset{k \in K}{U}}_{u \in F_{k}}\left\{\Gamma_{y}: u \in \Gamma_{y}\right\}\right\} \\
& =\underset{k \in K}{u}\left(\bigcap_{u \in F_{k}}\left\{\Delta \in M_{X}: u \in \Delta\right\}\right) \\
& =\bigcup_{k \in K}\left(\prod_{u \in F_{k}} S(\{u\})\right) \\
& =\bigcup_{k \in K}\left(S\left(F_{k}\right)\right) .
\end{aligned}
$$

Since each. $F_{k}$ is finite and $F_{k} \subseteq X$, we have that $S\left(F_{k}\right)$ is a member of the base for $\left(M_{X}, \tau\right)$, and so $\theta[P]$ is open in $\left(M_{X}, \tau\right)$.

This shows that $Y$ is homeomorphic to the dual space of a closure 
algebra $(X, C)$ with a countable carrier $X$. It remains to be shown that $(X, C)$ is separated. Suppose that $A_{f} \subseteq \Delta \in M_{X}$, and so $\Delta \in S\left(A_{f}\right) \subseteq M_{X}$. Then since $\left(M_{X}, \tau\right)$ is regular, there is some open neighbourhood 0 such that $\Delta \in 0 \subseteq \overline{0} \subseteq S\left(A_{f}\right)$, and hence some $S\left(A_{g}\right)$

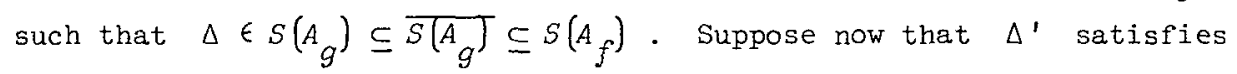
$A_{f} \notin \Delta^{\prime}$; then $\Delta^{\prime} \notin S\left(A_{f}\right)$ and so $\Delta^{\prime} \notin \overline{S\left(A_{g}\right)}$. Hence there is some $S\left(A_{g}^{\prime}\right)$ with $\Delta^{\prime} \in S\left(A_{g}^{\prime}\right)$ and $S\left(A_{g}\right) \cap S\left(A_{g}^{\prime}\right)=\emptyset$; that is, $S\left(A_{g} \cup A_{g}^{\prime}\right)=\emptyset$ and so for each $y \in Y, A_{g} \cup A_{g}^{\prime} \Phi_{y}$. But now for each $y \in Y$, $y \notin\left\{u: u \in A_{g} \cup A_{g}^{\prime}\right\} ;$ that is,

$$
\cap\left\{u: u \in A_{g}^{\prime} \cup A_{g}^{\prime}\right\}=\emptyset
$$

and

$$
C\left(A_{g} \cup A_{g}^{\prime}\right)=\left\{v: v \underline{z} \cap\left\{u: u \in A_{g} \cup A_{g}^{\prime}\right\}\right\}=X
$$

Thus $A_{g}^{\prime} \subseteq \Delta^{\prime}$ and $A_{g} \cup A_{g}^{\prime}$ is inconsistent. This shows $(X, C)$ to be separated. //

In order to prove analogues of these theorems for the case of an arbitrary metric space, we need the following definition and result from general topology (both given in ArchangelskiT [1]).

DEFINITION. The base of a topological space is called regular if, for each point $x$ and each neighbourhood $U$ of $x$, there exists a neighbourhood $V$ of $x$ such that only finitely many members of the base intersect both $V$ and the complement of $U$.

ARCHANGELSKIY'S FIRST METRIZATION THEOREM. A $T_{1}$-space is metrizable if and only if it has a regular base.

THEOREM 3. If $(X, C)$ is a strongly separated closure algebra then the dual space, $\left(M_{X}, \tau\right)$ is a metric space.

Proof. From Logan [3] we have that $\left(M_{X}, \tau\right)$ is a $T_{1}$-space, so that by ArchangelskiY's Theorem we need only show that the base of $\left(M_{X}, \tau\right)$ is regular. Suppose that $\Delta \in H_{X}$ and $U$ is a neighbourhood of $\Delta$; then 
there is some $S\left(A_{f}\right) \in B$ with $\Delta \in S\left(A_{f}\right) \subseteq U$; that is, with $A_{f} \subseteq \Delta$ and $M_{X} \backslash S\left(A_{f}\right) \supseteq M_{X} \backslash U$. Since $(X, C)$ is strongly separated we may choose $A_{g} \subseteq \Delta$ satisfying the conditions of Definition (II). Put $V=S\left(A_{g}\right)$; since $A_{g} \subseteq \Delta, V$ is a neighbourhood of $\Delta$, and if $S\left(A_{h}\right) \in \beta$ is such that $S\left(A_{h}\right) \cap M_{X} \backslash U \neq \emptyset, S\left(A_{h}\right) \cap V \neq \varnothing$, then

$$
\begin{gathered}
S\left(A_{h}\right) \cap M_{X} \backslash S\left(A_{f}\right) \neq \emptyset \text { and } S\left(A_{h}\right) \cap S\left(A_{g}\right) \neq \emptyset \\
\Rightarrow \text { for some } \Delta_{1}, \Delta_{1} \in S\left(A_{h}\right), \Delta_{1} \notin S\left(A_{f}\right) \text {, and } S\left(A_{h} \cup A_{g}\right) \neq \emptyset, \\
\Rightarrow \text { for some } \Delta_{1}, A_{h} \subseteq \Delta_{1}, A_{f} \pm \Delta_{1}, \text { and } C\left(A_{h} \cup A_{g}\right) \neq X .
\end{gathered}
$$

Now since $(X, C)$ is strongly separated there are onily finitely many such sets $A_{h}$, and so there are only finitely many members of the base $S\left(A_{h}\right)$ that intersect both $V$ and $M_{X} \backslash U$. We may infer that $\left(M_{X}, \tau\right)$ is metrizable. //

THEOREM 4. Every metric space is homeomorphic to the dual space of a strongly separated closure algebra.

Proof. Let $Y$ be a metric space; then $Y$ is a $T_{1}$-space with a regular base. Take $X$ as a subbase for the regular base of the topology on $Y$, and notice that in the proof of Theorem 2 the only property required to construct $(X, C)$ and $\left(M_{X}, \tau\right)$ so that $\left(M_{X}, \tau\right)$ is homeomorphic to $Y$, is that $Y$ be $T_{I}$. If these constructions are made then the only other requirement is to show that $(X, C)$ is strongly separated. Suppose that $\Delta \in M_{X}$ and $A_{f} \subseteq \Delta$; then $\Delta \in S\left(A_{f}\right)$ and $S\left(A_{f}\right) \in B$ is a neighbourhood of $\Delta$. Since the base is regular there is some neighbourhood $V$ of $\Delta$ with only finitely many members of $\beta$ intersecting both $V$ and $M_{X} \backslash S\left(A_{f}\right)$. Since $V$ is a neighbourhood of $\Delta$ we may choose some $S\left(A_{g}\right) \in B$ with $\Delta \in S\left(A_{g}\right) \subseteq V$, and now there are only finitely many $S\left(A_{h}\right) \in B$ with $S\left(A_{h}\right) \cap S\left(A_{g}\right) \neq \emptyset$ and $S\left(A_{h}\right) \cap M_{X} \backslash S\left(A_{f}\right) \neq \varnothing$; that is, with $S\left(A_{h} \cup A_{g}\right) \neq \varnothing$ and with some $\Delta_{1} \in M_{X}$ satisfying $\Delta_{1} \in S\left(A_{h}\right), \Delta_{1} \notin S\left(A_{f}\right)$. This being the case there 


$$
\text { Representation of metric spaces }
$$

are only finitely many sets $A_{h}$ with $C\left(A_{h} \cup A_{g}\right) \neq X$ and $A_{h} \leqq \Delta$ for some $\Delta_{1}$ with $A_{f} \notin \Delta_{1}$. This shows $(X, C)$ to be strongly separated. //

\section{References}

[1] А. Архангельсний [A. Archangielskiт], "О метризацин топологичесних пространств" [On the metrization of topological spaces], BuZl. Acad. Polon. Sci. Sér. Sci. Math. Astronom. Phys. 8 (1960), 589-595.

[2] John L. Kelley, General topology (Van Nostrand, Toronto, New York, London, 1955. Reprinted: Graduate Texts in Mathematics, 27. Springer-Verlag, New York, Heidelberg, Berlin, [1975]).

[3] G.J. Logan, "Closure algebras and $\mathrm{T}_{1}$-spaces", 2. Math. Logik Grundlag. Math. 23 (1977), 91-92.

[4] G.J. Logan, "Closure algebras and boolean algebras", Z. Math. Logik Grundlag. Math. 23 (1977), 93-96.

Department of Applied Sciences,

Christchurch Technical Institute,

Christchurch,

New Zealand. 